\title{
Leg Pain in a Patient with Myasthenia Gravis
}

\author{
Nachiket Patel, $M D^{7}$, Sarah M. Baker, BSN, RN², and J. Americo Fernandes Filho, MD ${ }^{3}$ \\ ${ }^{1}$ Internal Medicine Division, Department of Medicine, Creighton University School of Medicine, Omaha, NE, USA; ${ }^{2}$ Alegent-Creighton Health, \\ Creighton University Medical Center, Omaha, NE, USA; ${ }^{3}$ VA Nebraska-Western lowa Health Care System, Department of Neurological \\ Sciences, University of Nebraska Medical Center, Omaha, NE, USA.
}

KEY WORDS: Nocardia; myasthenia gravis; prednisone; immunocompromised.

J Gen Intern Med 28(11): 1523

DOI: $10.1007 / \mathrm{s} 11606-013-2445-\mathrm{x}$

(c) Society of General Internal Medicine 2013

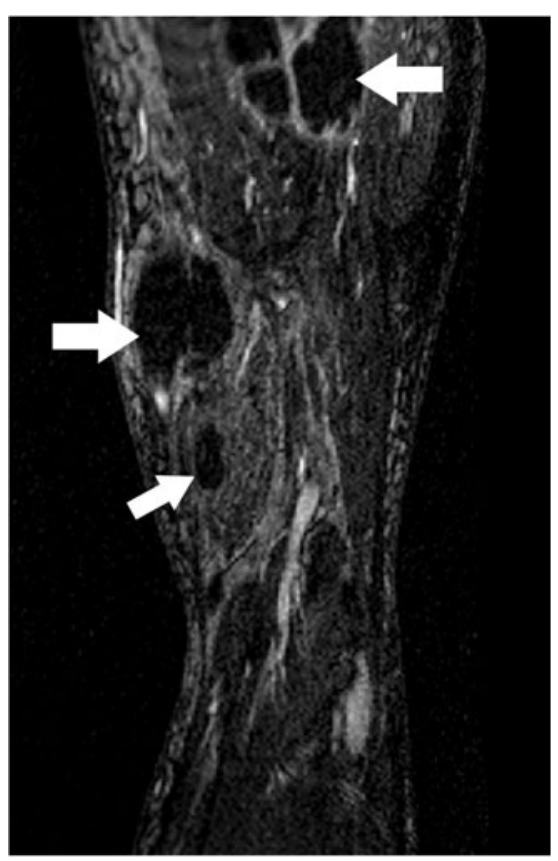

Figure 1. MRI of the left leg demonstrating multifocal abscesses in multiple muscular beds.

A 79-year-old male with myasthenia gravis on chronic prednisone therapy presented with progressive left leg pain and swelling and nonproductive cough for one month. Physical examination was significant for multiple, irregular, tender areas of induration in his bilateral lower extremities without overlying erythema. Laboratory data were significant for a white cell count of $12,400 / \mathrm{cmm}$.
MRI showed multifocal abscesses in his bilateral lower extremities within the muscle beds, which were more significant on the left (Fig. 1). He underwent ultrasoundguided drainage of the large abscesses. Cultures grew Nocardia pseudobrasiliensis sensitive to moxifloxacin. CT chest showed bilateral pleural effusions and ground-glass opacities in the right upper lobe, suggesting pulmonary nocardiosis with dissemination secondary to immunosuppression from chronic steroid use. The patient was started on moxifloxacin with a plan to treat for 12 months.

The majority of patients with nocardial infection are immunocompromised; the most common causes are glucocorticoid therapy, malignancy, organ transplantation, and HIV infection. Disseminated nocardial infection typically presents with deep abscess especially in the lower extremities or CNS. ${ }^{1}$ Treatment requires a long course of therapy: 6 months for pulmonary or systemic nocardiosis and at least 12 months for immunocompromised patients. ${ }^{2}$

Suspect norcardiosis in patients with CNS, soft tissue, or cutaneous lesions and a concurrent pulmonary process.

Financial Disclosure: The authors have no relevant funding to declare.

Conflict of Interest: The authors declare that they do not have a conflict of interest.

Corresponding Author: Nachiket Patel, MD; Internal Medicine Division, Department of Medicine, Creighton University School of Medicine, $601 \mathrm{~N}$ 30th Street, Suite 5850, Omaha, NE 68131, USA (e-mail: nachiketjpatel@gmail.com).

\section{REFERENCES}

1. Lederman ER, Crum NF. A case series and focused review of nocardiosis: clinical and microbiologic aspects. Med. (Baltimore). 2004;83(5):300-13.

2. Agterof MJ, van der Bruggen T, Tersmette M, et al. Nocardiosis: a case series and a mini review of clinical and microbiological features. Neth $\mathrm{J}$ Med. 2007;65(6): 199-202. 